\title{
AVALIAÇÃO DA FUNÇÃO GLOMERULAR DE CÃES SADIOS E NEFROPATAS SOB ESTIMULAÇÃO DOPAMINÉRGICA
}

\author{
Alexandre Martini de Brum ${ }^{1}$, Marileda Bonafim Carvalho ${ }^{1}$, Marcy Lancia Pereira ${ }^{1}$ \\ 1 Universidade Estadual Paulista (UNESP) - Campus de Jaboticabal - alexmbrum@bol.com.br
}

\begin{abstract}
RESUMO: A dopamina é um composto endógeno amplamente utilizado em terapia intensiva. Possui um amplo espectro de ações, tanto sobre o sistema cardiovascular como urinário. Aumento da taxa de filtração glomerular, do fluxo sangüíneo renal e excreção fracionada de sódio e fósforo são efeitos renais esperados em indivíduos normais, porém são pouco explorados na medicina veterinária. Este estudo foi conduzido com o propósito de avaliar a função glomerular de cães nefropatas crônicos submetidos à infusão contínua de dopamina. Diferentes doses de dopamina foram administradas em cães nefropatas. Avaliações laboratoriais de clearance de creatinina e a razão proteína/creatinina urinária foram realizadas durante e após os tratamentos. O clearance de creatinina apresentou aumento dose-dependente nos cães sadios. Em cães nefropatas, a dose de $1 \mu \mathrm{g} / \mathrm{kg} / \mathrm{min}$ aumentou discretamente a TFG, sem modificar a U-P/C e pressão arterial sistêmica, enquanto a dose de $3 \mu \mathrm{g} / \mathrm{kg} / \mathrm{min}$ promoveu aumento da excreção urinária de proteínas.
\end{abstract}

Palavras-chave: cão; dopamina; insuficiência renal crônica; proteinúria; taxa de filtração glomerular

\section{EVALUATION OF GLOMERULAR FUNCTION IN HEALTHY AND NEPHROPATHIC DOGS UNDER DOPAMINERGIC STIMULATION}

\begin{abstract}
Dopamine is an endogenous compound widely used in intensive care. It has a broad spectrum of action, on the cardiovascular system and urinary tract. Increased glomerular filtration rate, renal blood flow and fractional excretion of sodium and phosphorus are expected renal effects in normal individuals, but are poorly explored in veterinary medicine. This study was conducted to evaluate the glomerular function of dogs with renal disease submitted to continuous infusion of dopamine. Different doses of dopamine were administered in healthy and nephropathic dogs. Laboratory evaluations of creatinine clearance and urinary protein/creatinine ratio were performed during and after treatments. Creatinine clearance showed dose-dependent increase in healthy dogs. In dogs with renal disease, the dose of $1 \mu \mathrm{g} / \mathrm{kg} / \mathrm{min}$ GFR increased slightly, without changing the urine $\mathrm{P} / \mathrm{C}$ and blood pressure, while the dose of $3 \mu \mathrm{g} / \mathrm{kg} / \mathrm{min}$ increased urinary protein excretion.
\end{abstract}

Key Words: dog; chronic renal failure; dopamine; glomerular filtration rate; proteinuria 


\section{INTRODUÇÃO}

A dopamina é uma catecolamina endógena, precursora imediata da norepinefrina que apresenta a capacidade de estimular diferentes receptores dopaminérgicos e adrenérgicos de forma dose-dependente. Em doses baixas, interage predominantemente com receptores dopaminérgicos. Estes receptores são classificados em dois grupos: receptores semelhantes ao D1 (D1 e D5) e semelhantes ao D2 (D2, D3 e D4), que pertencem à superfamília de receptores acoplados a proteína-G (Hussain e Lokhandwala, 2003). A estimulação dos receptores D1 causa ativação da adenilato ciclase e aumento da concentração intracelular do segundo mensageiro AMPc, induzindo hipotensão e aumento de fluxo sanguíneo para os rins, cérebro, vasos mesentéricos e coronárias, além de diurese e natriurese (LeClair et al., 1998). A ação da estimulação D2 nos rins ainda é discutida. Apesar de estudos sugerirem que a natriurese deva-se à estimulação simultânea de receptores D1 e D2, alguns trabalhos demonstram que agonistas D2 causam antidiurese e antinatriurese (Hussain e Lokhandwala, 2003). Além disso, a estimulação deste receptor contribui para a hiperfiltração induzida por aminoácidos. Apesar do mecanismo exato de ação não estar esclarecido, acredita-se que seja através de estimulação neuronal (Luippold e Mühlbauer, 1998).

McDonald et al. (1964) demonstraram que a dopamina aumenta o fluxo sanguíneo renal em humanos por diminuição da resistência vascular renal. O mesmo efeito foi observado em cães, nos quais a dose de $1,2 \mu \mathrm{g} / \mathrm{kg} / \mathrm{min}$ aumentou em $38 \%$ o fluxo sanguíneo renal, sem alterar a pressão sanguínea arterial (McNay et al., 1965). Baixas doses de dopamina estimulam recepto- res D1 no leito vascular renal, induzindo a vasodilatação e aumento do fluxo sanguíneo renal (Tobata et al., 2004), mas doses altas, capazes de estimular receptores a-adrenérgicos, levam a vasoconstrição renal e de outros efeitos vasculares (Furukawa et al., 2002). Enquanto a estimulação de receptores D1 causa vasodilatação de forma direta, mediada pela via AMPc/PKA, a ação dos receptores tipo D2 é um pouco mais complexa. Estes receptores podem agir de forma indireta ou direta sobre a musculatura lisa vascular. De acordo com McDonald et al. (1964), o aumento no fluxo sanguíneo renal parece ser responsável pelo aumento na TFG, mas o aumento do débito cardíaco também desempenha um papel muito importante sobre este evento. A dopamina também é capaz de induzir aumento na TFG por outros mecanismos.

A estimativa da TFG pode ser realizada através do clearance de creatinina (Finco, 1971; Boveé e Joice, 1979), para isso os testes de depuração de creatinina endógena de 24 horas e de 20 minutos são empregados em pequenos animais (Finco, 1995a). Este método permite identificar precocemente animais com diminuição de função renal, mesmo que não apresentem azotemia (Boveé e Joice, 1979). Outra forma de avaliar a função renal é através da estimativa de perda de proteínas pela urina. A avaliação da excreção de proteínas urinárias em 24 horas e a razão proteínas-creatinina urinária $(\mathrm{U}: \mathrm{P} / \mathrm{C})$ são os métodos utilizados para determinar a perda renal de proteínas. O segundo método possui a vantagem de ser mais prático e rápido para detecção de proteinúria. Valores de $\mathrm{U}: \mathrm{P} / \mathrm{C}$ superiores a 0,5 podem indicar perdas renais de proteínas. Valores discretamente aumentados sugerem lesão tubular renal, uma vez que as proteínas normalmente filtradas não são 
reabsorvidas no túbulo contorcido proximal, enquanto valores moderadamente a gravemente aumentados sugerem lesão glomerular, pois, nestes casos, os glomérulos perderam sua capacidade de permeabilidade seletiva, permitindo a passagem de grande quantidade de proteínas (Finco, 1995b).

$\mathrm{Na}$ doença renal crônica, à medida que ocorre perda da capacidade funcional, os mecanismos compensatórios tentam aumentar a taxa de filtração glomerular. A constrição da arteríola eferente aumenta a pressão no glomérulo, aumentando, consequentemente, a taxa de filtração glomerular. Entretanto, este mecanismo acarreta, a longo prazo, destruição dos glomérulos. O arsenal terapêutico disponível para controlar este problema envolve dilatadores arteriais. Entre eles, os inibidores da enzima conversora da angiotensina (Gonin-Jmaa e Senior, 1995).

O objetivo do presente estudo foi avaliar a ação vasodilatadora renal da dopamina em cães nefropatas. Para tal, cães sadios e portadores de doença renal crônica foram submetidos a diferentes doses de dopamina para avaliação da função glomerular.

\section{MATERIAL E MÉTODOS}

Foram utilizados dois grupos de cães, um composto por cinco cães sadios (GS) e o outro por quatro nefropatas (GN). Foi realizada avaliação de perfil bioquímico (creatinina e uréia séricas), urinálise, razão proteína/creatinina urinária e clearance de creatinina. Os cães que não apresentaram alterações na avaliação inicial e cujo valor de clearance de creatinina era normal constituíram o GS. Os cães com nefropatia e valores de clearance de creatinina inferiores aos valores normais, azotêmicos ou não e sem manifestações clínicas de outras doenças, compuseram o GN. Segundo
Carneiro (2002), o valor de clearance de creatinina estimados pela técnica de 20 em duplicata para cães sadios deve ser superior a $1,45 \mathrm{~mL} / \mathrm{min} / \mathrm{kg}$.

\section{Protocolo experimental}

Os dois grupos foram submetidos à avaliação basal (controle negativo) e a outras seis avaliações para testar os efeitos de dois protocolos de infusão de dopamina em solução de $\mathrm{NaCl}$ a $0,9 \%$. Para cada um dos testes, a dopamina foi diluída em solução de $\mathrm{NaCl}$ a $0,9 \%$ de modo que fossem obtidas as taxas de infusão do fármaco $(1 \mu \mathrm{g} / \mathrm{kg} / \mathrm{min}$ e $3 \mu \mathrm{g} / \mathrm{kg} / \mathrm{min}$ ) e mantida a velocidade de administração do solvente (2mL/kg/hora). As avaliações incluíram o controle positivo (durante e 30 minutos após infusão de solução de $\mathrm{NaCl}$ a $0,9 \%$ ), o primeiro teste (durante e 30 minutos após infusão de dopamina na dose de $1 \mu \mathrm{g} / \mathrm{kg} / \mathrm{min}$ ) e o segundo teste (durante e 30 minutos após infusão de dopamina na dose de $3 \mu \mathrm{g} / \mathrm{kg} / \mathrm{min})$. A solução de $\mathrm{NaCl}$ a $0,9 \%$ foi administrada na taxa de infusão aproximada de $2 \mathrm{~mL} / \mathrm{kg} /$ hora. Em cada avaliação foi aferida a pressão arterial sistêmica, relação proteína-creatinina urinária e o clearance de creatinina. Os animais permaneciam na infusão por aproximadamente cem minutos, com uma pequena variação, conforme a dificuldade na realização das avaliações. As avaliações de cada um dos animais estudados, após o controle negativo, foram realizadas em intervalos de 24 horas.

A avaliação do clearance de creatinina $(\mathrm{Ccr})$ foi realizada através da técnica de 20 minutos em duplicata (Finco, 1995a) e adaptado por Carneiro (2002).

A avaliação da relação proteínas/creatinina urinária $(\mathrm{U}: \mathrm{P} / \mathrm{C})$ foi obtida através da divisão do valor da concentração urinária de proteínas totais pelo valor da concentração de creatinina urinária.

Foram realizadas dosagens de 
creatinina nas soluções e nos soros obtidos, para avaliação do clearance de creatinina. A creatinina foi dosada pelo método da reação de picrato em meio alcalino (kit Creatinina K, Labtest Diagnóstica). Na amostra de urina, diluída em 1:5, a creatinina foi dosada conforme descrito anteriormente e a proteína (kit Sensiprot, Labtest Diagnóstica), pelo método de vermelho de pirogalol, por colorimetria e com leitura em aparelho semi-automático (Labquest, Labtest Diagnóstica).

A pressão arterial foi obtida por meio de mensuração não-invasiva com aparelho oscilométrico (Dixtal ${ }^{\circledR}$ modelo DX2710). Foram realizadas cinco determinações e os valores limítrofes superiores e inferiores descartados para a obtenção de uma média mais acurada.

Os dados obtidos (razão proteína/creatinina urinária, clearance de creatinina e valores de pressão arterial sistêmica) foram submetidos à análise de variância, seguida pelo teste de Tukey, utilizado para comparação de médias entre os diferentes tratamentos dentro do mesmo grupo. Os dados das sessões um e dois, de cada tratamento, foram submetidos ao teste $t$ pareado. $O$ teste $t$ de Student foi utilizado para comparação de médias, entre grupos, obtidas durante cada tratamento. Essas análises foram realizadas pelo programa SigmaStat for Windows $\AA^{\circledR}$.

\section{RESULTADOS}

Os valores médios da relação proteína/creatinina urinária $(\mathrm{U}-\mathrm{P} / \mathrm{C})$ e clearance de creatinina (Ccr) dos cães sadios (GS) e dos nefropatas (GN) estão apresentados na Tabela 1.

A U-P/C dos cães sadios diminuiu com a administração de dopamina, porém não houve diferença significativa. Houve aumento da U-P/C na avaliação após 30 minutos do término da infusão em todos tratamentos, alcançando significância $\quad(p \leq 0,05) \quad$ quando considerado os valores durante e após a infusão da dose de $1 \mu \mathrm{g} / \mathrm{kg} / \mathrm{min}$ (Tabela 1 e Figura 1). A U-P/C dos cães nefropatas variou entre os indivíduos, no entanto, não foram identificadas alterações significativas relacionadas às infusões de dopamina (Tabela 1 e Figura 1). Os cães nefropatas 3 e 4 apresentaram aumento dos valores de U-P/C superiores a 1,0 durante a infusão de dopamina na dose de $3 \mu \mathrm{g} / \mathrm{kg} / \mathrm{min}$. O cão 3 já havia apresentado aumento neste parâmetro de magnitude semelhante durante a infusão de $1 \mu \mathrm{g} / \mathrm{kg} / \mathrm{min}$ de dopamina (Figura 2).

O Ccr aumentou significativamente $(p \leq 0,05)$ durante a infusão de dopamina nos animais sadios, sem diferenças nas respostas de cada uma das doses testadas. Tal efeito desapareceu no período pós-infusão (Tabela 1 e Figura 1). A infusão de dopamina nos cães nefropatas promoveu aumento do clearance de creatinina, porém sem diferença significativa. No período pósinfusão, as médias permaneceram superiores $(p \leq 0,05)$ quando comparadas com o controle. Todos os cães com doença renal apresentaram aumento do Ccr durante infusão das diferentes doses de dopamina (Tabela 1 e Figura 1). Nos cães 1, 2 e 3 do GN, durante a infusão de dopamina na dose de $1 \mu \mathrm{g} / \mathrm{kg} / \mathrm{min}$, os Ccr atingiram valores considerados de referência para a espécie. Com a dose de $3 \mu \mathrm{g} / \mathrm{kg} / \mathrm{min}$, entretanto, os $\mathrm{Ccr}$ dos cãe nefropatas não atingiram a faixa de normalidade (Figura 2). As pressões arteriais sistólicas não apresentaram alterações significativas durante as infusões ou na comparação entre grupos.

\section{DISCUSSÃO}

O presente estudo demonstrou que a dopamina apresenta ações sobre a função glomerular de cães sadios e 
Tabela 1 - Médias, desvios-padrão dos valores U-P/C e Ccr obtidos durante e 30 minutos após infusão de solução de $\mathrm{NaCl} 0,9 \%$ e de dopamina nas taxas de $1 \mu \mathrm{g} / \mathrm{kg} / \mathrm{min}$ (DA 1) e $3 \mu \mathrm{g} / \mathrm{kg} / \mathrm{min}$ (DA 3) em cães sadios e nefropatas.

\begin{tabular}{|c|c|c|c|c|c|c|c|}
\hline \multicolumn{2}{|c|}{ Parâmetros } & \multicolumn{4}{|c|}{ Sadios } & \multicolumn{2}{|c|}{ Nefropatas } \\
\hline & & & Durante & Após & & Durante & Após \\
\hline & Basal & $0,5 \pm 0,2^{a}$ & & & $0,4 \pm 0,2^{a}$ & & \\
\hline \multirow[t]{4}{*}{ U-P/C } & $\mathrm{NaCl}$ & & $0,6 \pm 0,3^{\mathrm{Aa}}$ & $0,8 \pm 0,3^{\mathrm{Aa}}$ & & $0,6 \pm 0,2^{\mathrm{Aa}}$ & $0,6 \pm 0,3^{\mathrm{Aa}}$ \\
\hline & DA 1 & & $0,2 \pm 0,1^{\mathrm{Aa}}$ & $0,4 \pm 0,2^{\mathrm{Ba}}$ & & $0,6 \pm 0,5^{\mathrm{Aa}}$ & $0,8 \pm 0,3^{\mathrm{Aa}}$ \\
\hline & DA 3 & & $0,2 \pm 0,1^{\mathrm{Aa}}$ & $0,4 \pm 0,2^{\mathrm{Aa}}$ & & $0,8 \pm 0,5^{\mathrm{Aa}}$ & $0,9 \pm 0,7^{\mathrm{Aa}}$ \\
\hline & Basal & $1,9 \pm 0,2^{a}$ & & & $1,0 \pm 0,1^{a}$ & & \\
\hline Ccr & $\mathrm{NaCl}$ & & $1,8 \pm 0,2^{\mathrm{Aa}}$ & $1,8 \pm 0,2^{\mathrm{Aa}}$ & & $1,0 \pm 0,3^{\mathrm{Aa}}$ & $0,8 \pm 0,1^{\mathrm{Aa}}$ \\
\hline \multirow[t]{2}{*}{$\mathrm{mL} / \mathrm{min} / \mathrm{kg}$} & DA 1 & & $2,2 \pm 0,4^{\mathrm{Ab}}$ & $1,8 \pm 0,2^{\mathrm{Aa}}$ & & $1,5 \pm 0,4^{\mathrm{Aa}}$ & $1,3 \pm 0,1^{\mathrm{Aa}}$ \\
\hline & DA 3 & & $2,3 \pm 0,2^{\mathrm{Ab}}$ & $1,8 \pm 0,2^{\mathrm{Ba}}$ & & $1,2 \pm 0,1^{\mathrm{Aa}}$ & $1,0 \pm 0,1^{\mathrm{Ba}}$ \\
\hline
\end{tabular}

Médias seguidas pela mesma letra maiúscula, na mesma linha, dentro de cada grupo, não diferem entre si pelo teste de $t$ de Student. $(p>0,05)$

Médias seguida por mesma letra minúscula, na mesma coluna, dentro de cada parâmetro, não diferem entre si pelo teste de Tukey $(p>0,05)$.

nefropatas. Estas ações renais da dopamina já foram descritas por McDonald et al (1964) em humanos e por McNay et al (1965) em cães saudáveis. Até o momento, não há descrição disponível na literatura, sobre a ação de compostos dopaminérgicos na função renal de cães nefropatas crônicos.

Em relação aos parâmetros pesquisados, foi possível observar que a razão proteína/creatinina urinária (U$\mathrm{P} / \mathrm{C}$ ) apresentou diminuição durante as duas infusões de dopamina em cães saudáveis. Este resultado era esperado e ocorreu, provavelmente, pela dilatação da arteríola eferente renal por estimulação de receptores D1 com doses baixas de dopamina (Hammond e Cutler, 1989). A U-P/C é um parâmetro indicativo de lesão ou hipertensão glomerular (Finco, 1995b). A constrição da arteríola eferente renal geralmente está relacionada com o aumento da pressão intraglomerular e conseqüente proteinúria. Desta forma, medidas terapêuticas que promovem dilatação desta arteríola são importantes no retardo da progressão da doença renal crônica (Gonin-Jmaa e Senior, 1995). A observação deste fato justificou a utilização terapêutica de agonistas dopaminérgicos em pacientes humanos com insuficiência renal crônica, para impedir a progressão da lesão renal (Docci, et al., 1986). Entretanto, os cães com doença renal crônica, do presente estudo, apresentaram aumento da U$\mathrm{P} / \mathrm{C}$, principalmente na dose de $3 \mu \mathrm{g} / \mathrm{kg} / \mathrm{min}$. Mesmo que não significativo, sugere-se que este aumento pode ter importância clínica, principalmente com relação ao desencadeamento ou agravamento da síndrome nefrótica. Quando avaliados individualmente, dois cães nefropatas apresentaram valores de U-P/C superiores a 1,0 durante a infusão de dopamina na dose de $3 \mu \mathrm{g} / \mathrm{kg} / \mathrm{min}$ e um deles já havia apresentado aumento semelhante quando testada a taxa de infusão de $1 \mu \mathrm{g} / \mathrm{kg} / \mathrm{min}$. O aumento chegou a $298 \%$ quando comparado ao basal com um dos cães nefropatas. Pequenas alterações na hemodinâmica renal, como constrição da arteríola eferente e conseqüente hipertensão glomerular, podem estar relacionadas 
$\mathrm{U}: \mathrm{P} / \mathrm{C}$ A

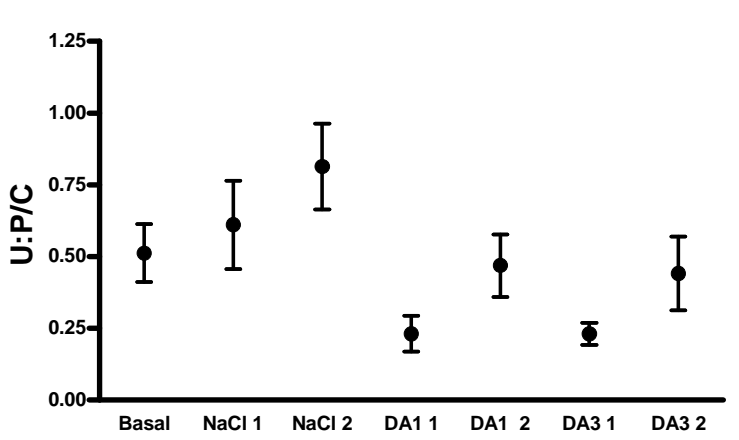

$\mathrm{U}: \mathrm{P} / \mathrm{C} \mathrm{B}$

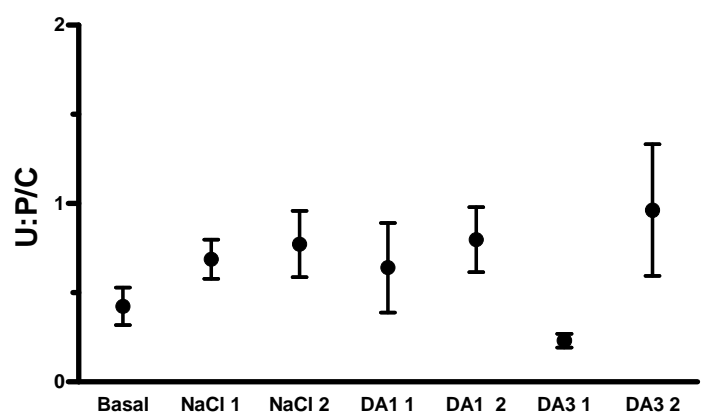

Ccr A

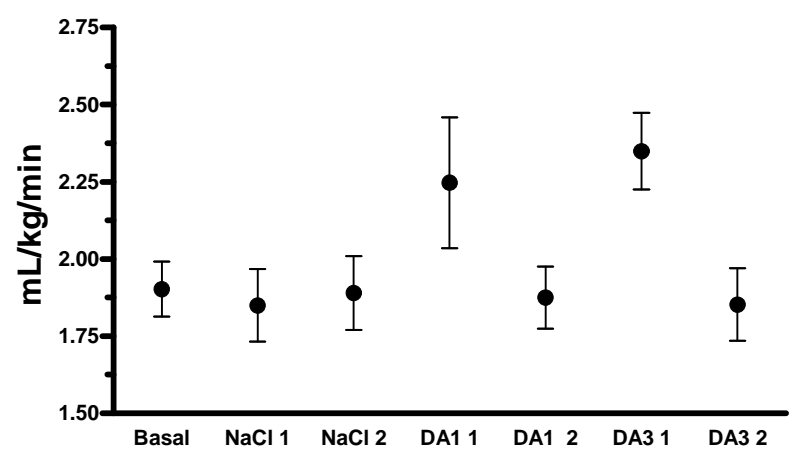

Ccr B

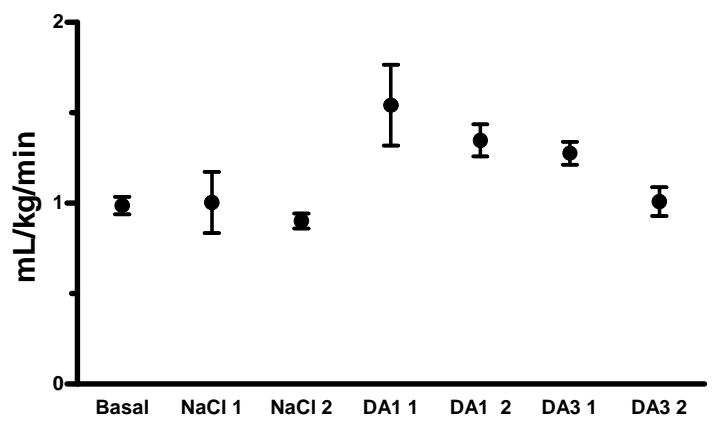

Figura 1 - Representações gráficas das médias dos valores e erro padrão da razão proteína/creatinina urinária (U-P/C) e clearance de creatinina (Ccr) obtidos durante (1) e 30 minutos após (2) infusão de solução de $\mathrm{NaCl} 0,9 \%$ e de dopamina, nas taxas de $1 \mu \mathrm{g} / \mathrm{kg} / \mathrm{min}$ (DA1) e $3 \mu \mathrm{g} / \mathrm{kg} / \mathrm{min}$ (DA3) em cães sadios (A) e nefropatas (B).

com o aumento de proteinúria, principalmente quando associadas às doenças túbulo-intersticiais, nas quais a reabsorção de proteínas está diminuída (Finco, 1995b). A hiperfiltração glomerular induzida por agonistas dopaminérgicos, associados ou não com sobrecarga protéica, também poderia ocasionar aumento da excreção renal de proteínas. A estimulação de receptores D2 é responsável por este fenômeno e a ativação destes, sobrepujando a ação de outros receptores dopaminérgicos, pode ocorrer devido maior sensibilidade destes ou por aumento na concentração local de dopamina (Luippold e Mühlbauer, 1998). Outra explicação possível seria a diminuição da afinidade dos receptores D1 no leito vascular renal, como ocorre em ratos com hipertensão sistêmica (Ladines, et al., 2001). A estimulação de receptores D3 pode causar constrição da arteríola eferente quando o tônus vascular em repouso está baixo (Zeng, et al., 2004), porém este apresenta-se elevado nos pacientes nefropatas (Hand, et al., 1999), tornando a vasoconstrição induzida por estes receptores pouco provável. Deve-se ressaltar que a pressão arterial sistêmica não apresentou diferença significativa e os valores mantiveram-se dentro do intervalo de referência para a espécie. Desta forma, aparentemente, o aumento da U-P/C observado não foi determinado por hipertensão.

O clearance de creatinina aumentou, de forma dose-dependente, 
$\mathrm{U}: \mathrm{P} / \mathrm{C}$

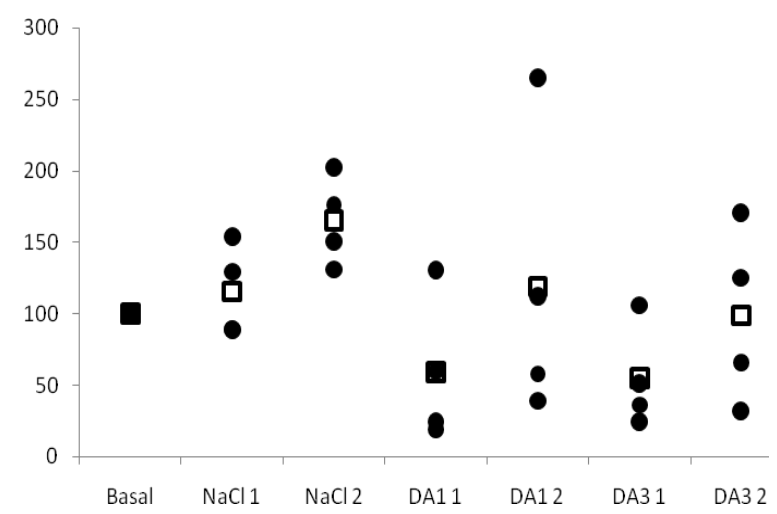

Ccr

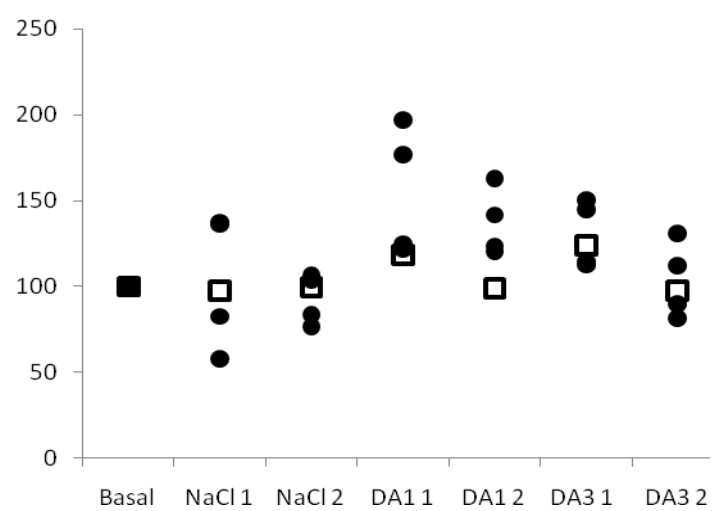

Figura 2 - Representações gráficas dos percentuais de variações, em relação aos valores basais (100\%) das médias dos cães sadios (quadrado vazado) e dos valores individuais dos cães nefropatas (círculos cheios) dos parâmetros razão proteína/creatinina urinária (U-P/C) e clearance de creatinina (Ccr) obtidos durante (1) e 30 minutos após (2) infusão de solução de $\mathrm{NaCl} 0,9 \%$ e de dopamina, nas taxas de $1 \mu \mathrm{g} / \mathrm{kg} / \mathrm{min}$ (DA1) e $3 \mu \mathrm{g} / \mathrm{kg} / \mathrm{min}$ (DA3).

nos cães sadios. A dose de $3 \mu \mathrm{g} / \mathrm{kg} / \mathrm{min}$ induz maior resposta diurética e natriurética (Hussain e Lokhandwala, 2003). No presente estudo, a dopamina determinou aumento do clearance de creatinina nos cães nefropatas, com maior magnitude na dose de $1 \mu \mathrm{g} / \mathrm{kg} / \mathrm{min}$. Segundo McDonald et al., (1964), o aumento na TFG está diretamente relacionado ao aumento do fluxo sangüíneo renal e, principalmente, ao débito cardíaco. O aumento apresentado pelos cães nefropatas ocorreu em uma dose que geralmente não causa aumento do débito cardíaco, porém este parâmetro não foi avaliado no presente estudo. Como o incremento da TFG ocorreu em conjunto com a elevação da U-P/C, a hiperfiltração glomerular induzida pela ativação de receptores D2 (Luippold e Mühlbauer, 1998), pode estar envolvida. A vasoconstrição da arteríola eferente, que poderia induzir aumento na TFG e U-P/C sem alterar o fluxo sanguíneo renal (Carey, 2001), parece pouco provável. Este fenômeno pode ser induzido pela estimulação de receptores D3, desde que o tônus vascular em repouso esteja baixo (Zeng, et al., 2004), o que não ocorre em pacientes com doença renal (Hand et al., 1999). Além disso, a estimulação de receptores dopaminérgicos $\mathrm{D} 1$ diminui a resposta renal à angiotensina II, que é um agente vasoconstritor renal conhecido (Barnett et al., 1986). Os limites terapêuticos da dopamina variam entre os indivíduos, tornando a resposta à terapia dopaminérgica muito particular (Gun e Mady, 1999). Três cães nefropatas utilizados neste estudo apresentaram aumento do $\mathrm{Ccr}$ durante a infusão da dose de $1 \mu \mathrm{g} / \mathrm{kg} / \mathrm{min}$, quando comparado aos cães saudáveis. Os mesmos cães, quando utilizada a dose de $3 \mu \mathrm{g} / \mathrm{kg} / \mathrm{min}$, não apresentaram aumento tão evidente. Esta diferença pode ser explicada quando se considera a resposta de cada paciente à dopamina. Dependendo da sensibilidade individual, doses maiores de dopamina podem estimular receptores $\beta$-adrenérgicos, aumentando 0 débito cardíaco $e$, consequentemente, a TFG. Tais receptores não estão envolvidos com vasodilatação renal e, assim, a magnitude da TFG deixa de ser tão significativa. A estimulação de receptores $\alpha$-adrenérgicos, se eventualmente tivesse ocorrido, causaria diminuição da TFG devido à 
vasoconstrição renal (Furukawa et al., 2002).

A dopamina exerce poucas alterações sobre a função glomerular de cães nefropatas. Doses maiores poderiam causar incremento na taxa de filtração glomerular, entretanto isso poderia ser prejudicial, pois doses mais altas podem estimular receptores D2 que induzem hiperfiltração glomerular (Luippold e Mühlbauer, 1998), evento importante na progressão da doença renal crônica. Além disso, um maior aporte de dopamina também pode estimular receptores a-adrenérgicos, que induzem vasoconstrição no leito renal e prejuízo para a função renal (Furukawa, et al., 2002). Na dose de $3 \mu \mathrm{g} / \mathrm{kg} / \mathrm{min}$ já foi notado aumento na perda renal de proteínas, evento importante na progressão da doença renal, possivelmente mediado pelo aumento da pressão glomerular (GoninJmaa e Senior, 1995). Na avaliação individual dos animais, alguns apresentaram elevações significativas na proteinúria, sugerindo que as alterações hemodinâmicas renais induzidas pela dopamina podem induzir a hiperfiltração glomerular (Luippold e Mühlbauer, 1998).

\section{CONCLUSÃO}

Os efeitos renais dopaminérgicos variam tanto em tipo quanto intensidade em função da dose administrada para cães sadios. Contudo, em indivíduos com nefropatias crônicas, independentemente da dose administrada, as alterações renais existentes limitam os tipos e intensidade das respostas. Nos cães nefropatas, a infusão de dopamina, na dose de $1 \mu \mathrm{g} / \mathrm{kg} / \mathrm{min}$, por período aproximado de cem minutos aumenta discretamente a TFG, sem modificar a U-P/C e pressão arterial sistêmica. A dose de $3 \mu \mathrm{g} / \mathrm{kg} / \mathrm{min}$ não aumenta a TFG em relação à dose de $1 \mu \mathrm{g} / \mathrm{kg} / \mathrm{min} \mathrm{e}$, ainda, promove aumento da excreção urinária de proteínas. Com base nestes achados, a dopamina deve ser usada com cautela em pacientes com doença renal crônica, principalmente em doses maiores, devido ao risco de aumentar a proteinúria dos pacientes.

\section{REFERÊNCIAS}

BARNETT, R.; SINGHAL. P.C.;

SCHARSCHMIDT, L.A. et al. Dopamine

Attenuates the contractile response to

angiotensin II in isolated rat glomeruli and

cultured mesangial cells. Circulation Research, v.59, p.529-533, 1986.

BOVÉE, K.C.; JOICE, B.A. Clinical evaluation of glomerular function: 24-hours creatinine clearance. Journal of American Veterinary Medical Association, v.174, n.5, p.488-491, 1979.

CAREY, R.M. Renal dopamine system: paracrine regulator of sodium homeostasis and blood pressure. Hipertension, v.38, p.297-302, 2001.

CARNEIRO, R.S. Contribuição ao estudo do clearance de creatinina e da excreção fracionada de sódio, potássio e uréia e determinação de valores de referência de indicadores de função renal. 2002.

Jaboticabal, 82f. Dissertação (Mestrado em Clínica Médica) - Curso de Pós-graduação em Medicina Veterinária, Universidade Estadual Paulista Júlio de Mesquita Filho.

DOCCI, D.; PISTOCCHI, E.; TURCI, F. et al. Effect of ibopamine on the progression of chronic renal failure. Clinical Nephrology, v.26, n.3, p.121-124, 1986.

FINCO, D.R. Simultaneous determination of phenolsulfonphthalein excretion and endogenous creatinine clearance in the normal dog. Journal of American Veterinary Medical Association, v.159, n.3, p.336-340, 1971.

FINCO, D.R. Evaluation of Renal Functions. In: OSBORNE, C.A.; FINCO, D.R. (Eds) Canine and Feline Nephrology and Urology.

Baltimore: Williams e Wilkins, p.29-46,1995a.

FINCO, D.R. Urinary Protein Loss. In: OSBORNE, C.A.; FINCO, D.R. (Eds) Canine and Feline Nephrology and Urology.

Baltimore: Williams e Wilkins, p.29-46,1995b.

FURUKAWA, S.; NAGASHIMA, Y.; HOSHI, K. et al. Effects of Dopamine Infusion on Cardiac and Renal Blood Flows in Dogs. The Journal of 
Veterinary Medical Science, v.64, n.1, p.41-44, 2002.

GONIN-JMAA, D.; SENIOR, D.F. The hyperfiltration theory: progression of chronic renal failure and the effects of diet in dogs. Journal of American Veterinary Medical Association, v.207, n.11, p.1411-1415, 1995.

GUN, C.; MADY, C. Inotrópicos Não-Digitálicos. In: BATLOUNI, M.; RAMIRES, J.A.F.

Farmacologia e Terapêutica Cardiovascular. São Paulo, Atheneu, 1999, p. 101-116.

HAMMOND, P.G.; CUTLER R.E. Dopamine, dopaminergic agents, and the kidney. Part I: Pharmacology, vascular e tubular effects.

Dialysis \& Transplantation. v.18, p.36-37, 1989.

HAND, M.F.; HAYNES, W.G.; WEBB, D.J. Reduced endogenous endothelin-1 mediated vascular tone in chronic renal failure. Kidney International, v.55, p.613-620, 1999.

HUSSAIN, T.; LOKHANDWALA, M.F. Renal Dopamine Receptor and Hypertension.

Experimental Biology and Medicine, v.228, p.134-142, 2003.

LADINES, C.A.; ZENG, C.; ASICO, L.D. et al. Impaired renal D1-like and D2-like dopamine receptors interaction in the spontaneously hypertensive rat. American Journal of Physiology: Regulatory, Integrative and Comparative Physiology, v.281, p.1071-1078, 2001.
LECLAIR, M. M.; BERNDT, T. J.; KNOX, F.G. Effects of renal interstitial infusion of $L$-dopa on sodium and phosphate excretions. Jounal of Laboratory and Clinical Medicine, v.132, n.4, p.308-312, 1998.

LUIPPOLD, G.; MÜHLBAUER, B. Dopamine D2 Receptors Mediate Glomerular Hyperfiltration Due To Amino Acids. Journal of Pharmacology and Experimental Therapeutics, v.286, p.1248-1252, 1998.

McDONALD, R.H.; GOLDBERG, L.I.; MCNAY, J.L. et al. Effects of Dopamine in Man: Augmentation of Sodium Excretion, Glomerular Filtration Rate, and Renal Plasma Flow. Journal of Clinical Investigation, v.43, n.6, p.11161124, 1964.

McNAY, J.L.; McDONALD, R.H.; GOLDBERG, L.I. Direct Renal Vasodilatation Produced by Dopamine in the Dog. Circulation Research, v.26, p.510-517, 1965.

TOBATA, D.; TAKAO, K.; MOCHIZUKI, M. et al. Effects of Dopamine, Dobutamine, Amrinone and Milrinone on Regional Blood Flow in Isoflurane Anesthetized Dogs. The Journal of Veterinary Medical Science, v.66, n.9, p.10971105, 2004.

ZENG, C.; WANG, D.; YANG, Z. et al. Dopamine $D 1$ receptor augmentation of $D 3$ receptor action in rat aortic or mesenteric vascular smooth muscles. Hypertension, v.43, p.673-679, 2004. 